\title{
Screening for cardio-metabolic risk factors in the Romanian cohort of HIV-positive patients
}

\author{
Anca Streinu-Cercel ${ }^{1,2^{*}}$, Oana Săndulescu ${ }^{1,2}$, Claudiu Mihai Șchiopu², Adrian Streinu-Cercel ${ }^{1,2}$ \\ From The 10th Edition of the Scientific Days of the National Institute for Infectious Diseases "Prof Dr Matei \\ Bals" \\ Bucharest, Romania. 15-17 October 2014
}

\section{Background}

HIV is recognized as an independent factor related to cardiovascular (CV) disease [1]. International guidelines recommend evaluation and adjustment of common cardiovascular risk factors for all HIV-positive patients. As antiretroviral (ARV) drugs associate different profiles regarding the CV risk [2], the therapeutic regimens need to be managed on a case-by-case basis [3,4]. The prevalence of CV disease appears to be higher in the HIV population, compared to the general population, and recent studies have demonstrated that the supplementary risk for acute myocardial infarction in HIV-infected patients is $75 \%$ [5].

\section{Methods}

We are currently performing a screening study in a cohort of HIV-infected patients to assess cardiovascular and metabolic involvement, through means of: echocardiography, intima-media thickness, Framingham score, serum lipid profile, DXA evaluation, as well as immune and virological evaluation.

\section{Results}

We present the results from a pilot project that included 100 patients from the Romanian HIV cohort. We evaluated 100 patients, with a mean age of 39.8 years old. The male-to-female ratio was 1.7:1. The mean CD4 count was 668 cells $/ \mathrm{cmm}$, with $5 \%$ of patients presenting CD4 cell counts below 200, 29\% between 200-500 and 66\% above 500 cells/cmm. The distribution of HIV-RNA was: undetectable (51\%), detectable, below 1,000 copies/mL (39\%), $1,000-10,000$ (4\%) and above 10,000 (6\%). Upon serum evaluation, $49 \%$ of patients had normal cholesterol levels (below $200 \mathrm{mg} / \mathrm{dL}$ ), 44\% had normal triglyceride values (below $150 \mathrm{mg} / \mathrm{dL}$ ), and 69\% had normal glycemic values.

\section{Conclusion}

These preliminary results warrant the continuation of this pilot study, with inclusion of a higher number of patients, in order to reach the project-specified target of enrollment.

\section{Acknowledgements \\ 1) Cardio-metabolic project - Merck Sharp \& Dohme. \\ 2) POSDRU/159/1.5/S/137390.}

\section{Authors' details}

${ }^{1}$ Carol Davila University of Medicine and Pharmacy, Bucharest, Romania. ${ }^{2}$ National Institute for Infectious Diseases "Prof. Dr. Matei Balş", Bucharest, Romania.

Published: 15 October 2014

\section{References}

1. Grunfeld C, Delaney JA, Wanke C, Currier JS, Scherzer R, Biggs ML, Tien PC, Shlipak MG, Sidney S, Polak JF, O'Leary D, Bacchetti P, Kronmal RA: Preclinical atherosclerosis due to HIV infection: carotid intima-medial thickness measurements from the FRAM study. AIDS 2009, 23:1841-9.

2. Daglan E, Yamin D, Manu B, Streinu-Cercel A: Cardiac involvement in HIVpositive patients. GERMS 2013, 3:8-13.

3. Guaraldi G: Evolving approaches and resources for clinical practice in the management of HIV infection in the HAART era. GERMS 2011, 1:6-8.

4. Errata. GERMS 2013, 3:67.

5. Triant VA: HIV infection and coronary heart disease: an intersection of epidemics. J Infect Dis 2012, 205(Suppl 3):S355-61.

doi:10.1186/1471-2334-14-S7-P61

Cite this article as: Streinu-Cercel et al.: Screening for cardio-metabolic risk factors in the Romanian cohort of HIV-positive patients. BMC Infectious Diseases 2014 14(Suppl 7):P61.

* Correspondence: anca_sc@yahoo.com

${ }^{1}$ Carol Davila University of Medicine and Pharmacy, Bucharest, Romania

Full list of author information is available at the end of the article 